\title{
Asynchronous Oscillations of Double-Mode and N-Phase in a Ring of Simultaneous Oscillators
}

\author{
Saori FUJIOKA ${ }^{\dagger}$, Yang YANG $^{\ddagger}$, Yoko UWATE ${ }^{\dagger}$ and Yoshifumi NISHIO ${ }^{\dagger}$ \\ $\dagger$ Dept. Electrical and Electronic Eng., Tokushima University \\ 2-1 Minami-Josanjima, Tokushima, Japan \\ Email: \{saori, uwate, nishio\}@ee.tokushima-u.ac.jp \\ $\ddagger$ The Institute of Artificial Intelligence and Robotics., Xi'an Jiaotong University \\ No. 28 Xianning-West-Road, Xi'an, China \\ Email: yyang@mail.xjtu.edu.cn
}

\begin{abstract}
In this study, a ring of coupled simultaneous oscillators with two $L C$ resonators is investigated. By computer simulations, asynchronous oscillations of doublemode and $N$-phase are confirmed to be stably generated. Careful observation reveals various interesting synchronization phenomena which have not reported before.
\end{abstract}

\section{Introduction}

In the natural fields, various synchronization phenomena exists. For example, firefly luminescence, swing of pendulums, cardiac heartbeat, and so on, are well known as synchronization phenomena. Oscillators containing a nonlinear resistor whose $v-i$ characteristics are described by fifth-power nonlinear characteristics are known to exhibit hard excitation [1][2]. Namely, the origin is asymptotically stable and an proper initial condition, which is larger than a critical value, is necessary to generate the oscillation. Such an oscillator is often called as hard oscillator or said to have hard nonlinearity.

Two identical oscillators with hard nonlinearities coupled by an inductor [3] are investigated by Datardina and Linkens. They have confirmed that nonresonant doublemode oscillations, which could not occur for the case of third-power nonlinearity, were stably excited in the coupled system. They have also confirmed that four different modes coexist for some range of parameter values; zero, two single-modes, and a double-mode.

In 1954, Schaffner reported that an oscillator with two degrees of freedom could oscillate simultaneously at two different frequencies when the nonlinear characteristics are described by a fifth-power polynomial function [4]. $\mathrm{Ku}-$ ramitsu also investigated the simultaneous oscillations for three or more degrees case theoretically and confirmed the generation of simultaneous oscillation with three frequencies by circuit experiments [5]. The simultaneous oscillations are definitely one of the most common nonlinear phenomena observed in various higher-dimensional systems in the natural science fields. However, after their pioneering works, as far as the authors know, there have not been many researches clarifying the basic mechanism of the simultaneous oscillations except [6][7].

In our past study, we investigated synchronization phenomena observed from two or more coupled hard oscillators [8]-[10]. In those studies, we could observe various interesting nonlinear phenomena including asynchronous double-mode oscillations and wave propagations.

In this study, we investigate a ring of simultaneous oscillators with two $L C$ resonators coupled by inductors. By computer simulations, asynchronous oscillations of double-mode and $N$-phase are confirmed to be stably generated. Careful observation reveals various interesting synchronization phenomena which have not reported before.

\section{Circuit Model}

The circuit model is shown in Fig. 1. In the circuit, $N$ simultaneous oscillators with two $L C$ resonators are coupled by inductors $L_{C}$ as a ring. Each simultaneous oscillator consists of a nonlinear negative resistor, whose $v-i$ characteristics are described by a fifth-power polynomial function as

$$
i_{R}(v)=g_{1} v-g_{3} v^{3}+g_{5} v^{5} \quad\left(g_{1}, g_{3}, g_{5}>0\right),
$$

and two resonators with different natural frequencies ( $\sqrt{L_{1} C_{1}}$ and $\sqrt{L_{2} C_{2}}$ ). The equations governing these coupled oscillators are described by the following differential equations.

$$
\left\{\begin{array}{l}
C_{1} \frac{d v_{j 1}}{d t}=-i_{j 1}-i_{R j}-i_{C j}+i_{C, j-1} \\
C_{2} \frac{d v_{j 2}}{d t}=-i_{j 2}-i_{R j}-i_{C j}+i_{C, j-1} \\
L_{1} \frac{d i_{j 1}}{d t}=v_{j 1} \\
L_{2} \frac{d i_{j 2}}{d t}=v_{j 2} \quad(j=1,2, \cdots, N),
\end{array}\right.
$$

where $i_{C 0}=i_{C N}$. The currents through the coupling inductors $i_{C j}$ are given as

$$
i_{C j}=\frac{L_{1}\left(i_{j 1}-i_{j+1,1}\right)+L_{2}\left(i_{j 2}-i_{j+1,2}\right)}{L_{C}}
$$


where $i_{N+1, k}=i_{1 k}(k=1,2)$. The currents through the nonlinear resistors $i_{R j}$ are given as

$$
i_{R j}=i_{R}\left(v_{j 1}+v_{j 2}\right)
$$

By using the following variables and parameters,

$$
\begin{aligned}
& v_{j k}=\sqrt[4]{\frac{g_{1}}{5 g_{5}}} x_{j k}, \quad i_{j k}=\sqrt[4]{\frac{g_{1}}{5 g_{5}}} \sqrt{\frac{C_{1}}{L_{1}}} y_{j k}, \\
& \alpha_{C}=\frac{C_{1}}{C_{2}}, \quad \alpha_{L}=\frac{L_{1}}{L_{2}}, \quad \gamma=\frac{L_{1}}{L_{C}}, \\
& \varepsilon=g_{1} \sqrt{\frac{L_{1}}{C_{1}}}, \quad \beta=\frac{3 g_{3}}{g_{1}} \sqrt{\frac{g_{1}}{5 g_{5}}}, \quad t=\sqrt{L_{1} C_{1}} \tau,
\end{aligned}
$$

the normalized circuit equations are given as follows.

$$
\left\{\begin{array}{l}
\frac{d x_{j 1}}{d \tau}=-y_{j 1}-f\left(x_{j 1}+x_{j 2}\right)-y_{C j}+y_{C, j-1} \\
\frac{d x_{j 2}}{d \tau}=\alpha_{C}\left\{-y_{j 2}-f\left(x_{j 1}+x_{j 2}\right)-y_{C j}+y_{C, j-1}\right\} \\
\frac{d y_{j 1}}{d \tau}=x_{j 1} \\
\frac{d y_{j 2}}{d \tau}=\alpha_{L} x_{j 2} \quad(j=1,2, \cdots, N),
\end{array}\right.
$$

where $y_{C 0}=y_{C N} \cdot y_{C j}$ corresponds to $i_{C j}$ and is given as

$$
y_{C j}=\gamma\left(y_{j 1}-y_{j+1,1}+\frac{y_{j 2}-y_{j+1,2}}{\alpha_{L}}\right)
$$

where $y_{N+1, k}=y_{1 k}(k=1,2)$. The nonlinear function $f(\cdot)$, which corresponds to the $v-i$ characteristics of the nonlinear resistors, is given as

$$
f(x)=\varepsilon\left(x-\frac{\beta}{3} x^{3}+\frac{1}{5} x^{5}\right) .
$$

\section{Simulation results for five coupled oscillators}

In this article, we show only several computer simulation results because of the limitation of page space. Firstly, the results obtained from 5 coupled oscillators are given in this section.

A typical example of the observed results is shown in Fig. 2. Figure 2(a) shows attractors observed at 10 resonators. Upper figures correspond to the attractors observed from upper resonators and they have a shape of torus, namely upper resonators exhibit double-mode oscillations. On the other hand, lower figures correspond to lower resonators and they seem to have single frequency. Figure 2(b) shows the phase relationship between horizontally adjacent resonators. Upper figures show that upper resonators are almost synchronized at in-phase. While lower figures show that lower resonators are also almost synchronized but with some phase differences. Figure 2(c)

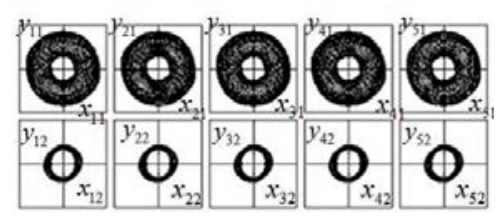

(a)

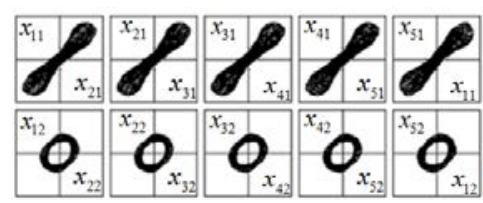

(b)

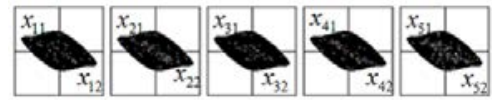

(c)

Figure 2: Typical example of obtained results from 5 coupled oscillators for $\alpha_{C}=0.8, \alpha_{L}=1.0, \gamma=0.3, \varepsilon=0.3$, and $\beta=3.5$. (a) Attractors observed at 10 resonators. Horizontal: $x_{j k}$. Vertical: $y_{j k}$. (b) Phase relationship between horizontally adjacent resonators. Horizontal: $x_{j+1, k}$. Vertical: $x_{j k}$. (c) Phase relationship between two resonators in each oscillator. Horizontal: $x_{j 2}$. Vertical: $x_{j 1}$.

shows the phase relationship between two resonators in each oscillator and that upper and lower resonators are not synchronized, namely asynchronous simultaneous oscillations occur.

Figure 3 shows the time waveforms of the voltages of 10 resonators. Figure 3(b) is the horizontally magnified figure of Fig. 3(a). From these figures we can observe that the upper resonators generate double-mode oscillations with their envelopes synchronized in 5-phase. Further, oscillations themselves of the upper resonators are synchronized in in-phase even though their amplitudes fluctuate periodically. Moreover, we can see that the lower resonators generate 5-phase synchronizations with almost constant amplitudes. We should also note that the fundamental oscillation frequencies of upper and lower resonators are different, namely they are asynchronous. As far as we know, this complicated phenomenon has never reported before. We believe that revealing the generation mechanism and conditions of such unknown phenomenon is important to understand highly nonlinear phonomena in natural fields and exploiting them for future engineering applications.

\subsection{Changing coupling strength}

Next, we investigate the effect of changing the coupling strength $\gamma$. The other parameters are fixed as the same in Figs. 2 and 3.

Figure 4 shows how the time waveforms of the voltages of 10 resonators change as $\gamma$ increases. The beat oscillations of the upper resonators become weaker and resulted in simple in-phase synchronizations as Fig. 4(c). While 


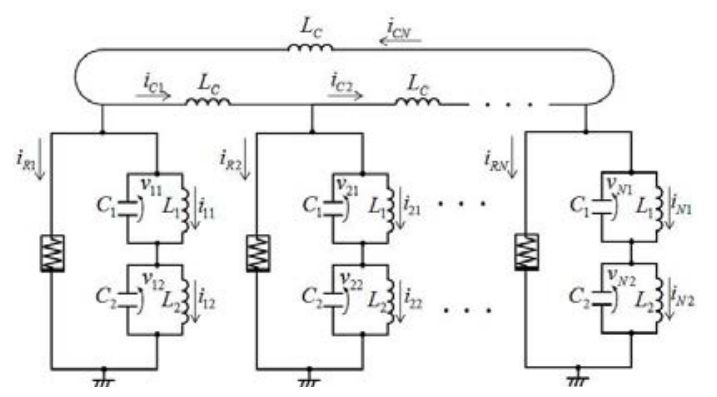

Figure 1: Ring of coupled simultaneous oscillators with two resonators.

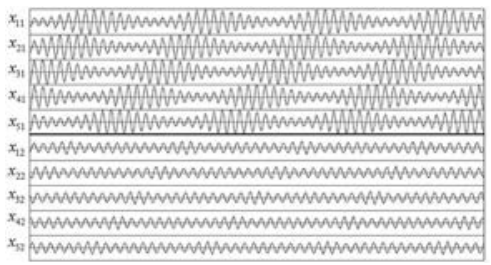

(a)

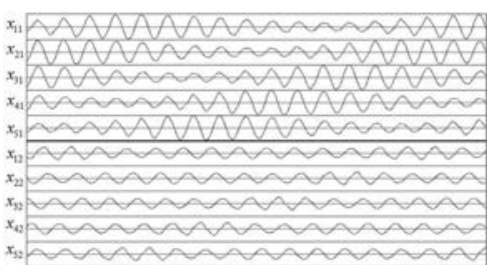

(b)

Figure 3: Time waveforms of the voltages of 10 resonators for $\alpha_{C}=0.8, \alpha_{L}=1.0, \gamma=0.3, \varepsilon=0.3$, and $\beta=3.5$.

the lower resonators become to show weak double-mode as Fig. 4(b), but resulted in almost oscillation-death as Fig. 4(c).

\subsection{Changing ratio of natural frequencies}

Next, we investigate the effect of changing the ratio of the natural frequencies of the resonators $\alpha_{C}$. The other parameters are fixed as the same in Figs. 2 and 3.

Figure 5 shows how the time waveforms of the voltages of 10 resonators change as $\alpha_{C}$ increases (equivalently $C_{2}$ becomes closer to $C_{1}$ ). The beat frequency of the upper resonators changes according to the ratio of natural frequencies. While the 5-phase oscillations of the lower resonators are not affected by the change of the ratio of the natural frequencies.

\section{Extension to larger rings of oscillators}

Finally, we show some simulation results observed from larger rings of oscillators.

Figure 6 shows a typical example of asynchronous oscillations observed from a ring of 7 coupled simultaneous oscillators. While, Fig. 7 shows time waveforms of the

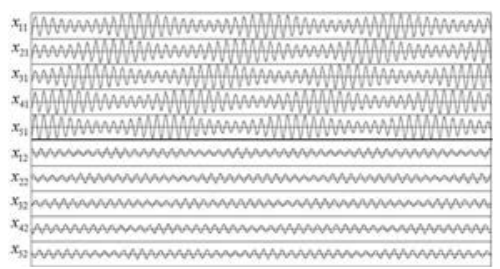

(a)

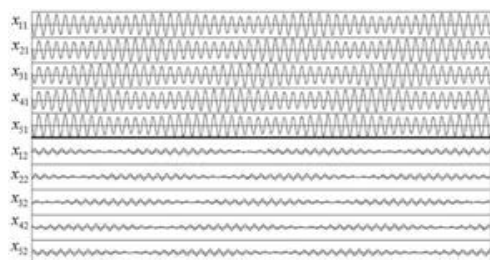

(b)

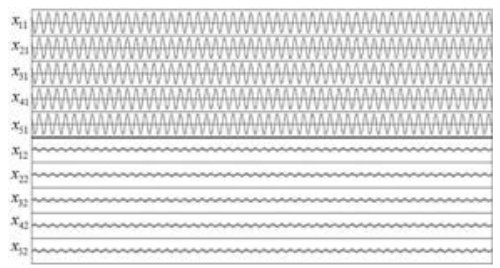

(c)

Figure 4: Time waveforms of the voltages of 10 resonators as changing coupling strength. (a) $\gamma=0.35$. (b) $\gamma=0.4$. (c) $\gamma=0.5$.

voltages observed from the ring of 15 simultaneous oscillators. As expected, we could observe asynchronous oscillations of double-mode in the upper resonators and $\mathrm{N}$-phase in the lower resonators from both rings. We also confirmed that the envelopes of the double-mode oscillations made 7phase and 15-phase synchronizations.

\section{Conclusions}

In this study, we investigated a ring of simultaneous oscillators with two $L C$ resonators coupled by inductors. By computer simulations, asynchronous oscillations of double-mode and $N$-phase were confirmed to be stably generated. We also investigated the effect of changing some important circuit parameters. 


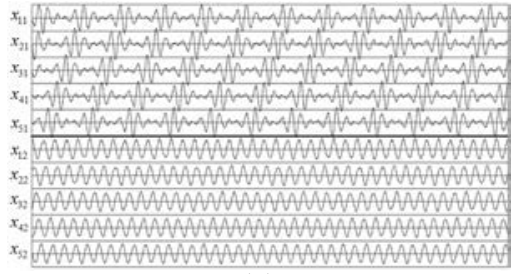

(a)

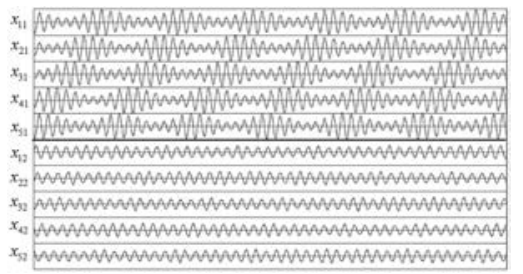

(b)

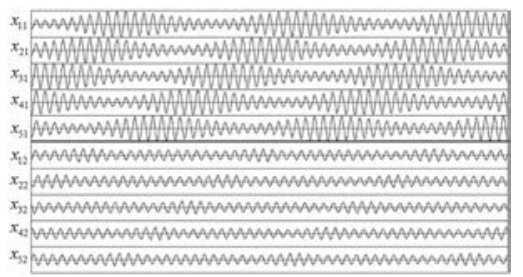

(c)

Figure 5: Time waveforms of the voltages of 10 resonators as changing ratio of natural frequencies. (a) $\alpha_{C}=0.5$. (b) $\alpha_{C}=0.7$. (c) $\alpha_{C}=0.85$.

We could not introduce some statistical analysis of the observed phenomena, because of the limitation of the page space. They will be presented at the conference, if accepted.

Our future works include the investigation for the case of three or more resonators in each oscillator and the circuit experiments.

\section{Acknowledgment}

This work was partly supported by JSPS Grant-in-Aid for Young Scientists 23700269.

\section{References}

[1] C. Hayashi, Nonlinear oscillations in physical systems, Princeton Univ. Press, p. 367, 1984

[2] V.I. Arnold, Geometrical methods in the theory of ordinary differential equa tions, Springer-Verlag, pp. 270-272, 1988.

[3] S.P. Datardina and D.A. Linkens, "Multimode oscillations in mutually coupled van der Pol type oscillators with fifth-power nonlinear characteristics," IEEE Trans. Circuits Syst., Vol. 25, No. 5, pp. 308-315, May 1978.

[4] J. Schaffner, "Simultaneous oscillations in oscillators," IRE Trans. Circuit Theory, Vol. 1, pp. 2-81, Jun. 1954

[5] M. Kuramitsu and F. Takase, "Averaged potential analysis of multi mode oscillators with hard operating conditions," IEICE Technical Report on NLP, Vol. 81, No. 13, pp. 1-10, Sep. 1981

[6] M. Matsuki and S. Mori, "Asynchronous simultaneous oscillations in negative resistance oscillatory circuit containing periodically operating analo switch," IEICE Technical Report on CAS, Vol. 101, pp. 81-87, Jun. 1993.

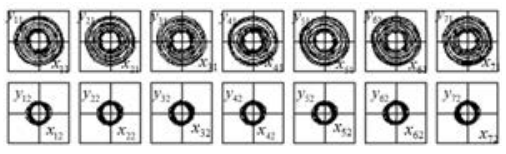

(a)

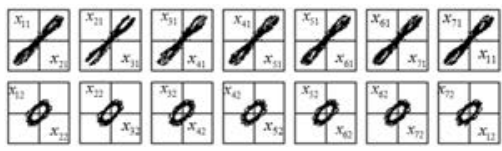

(b)

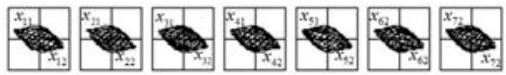

(c)

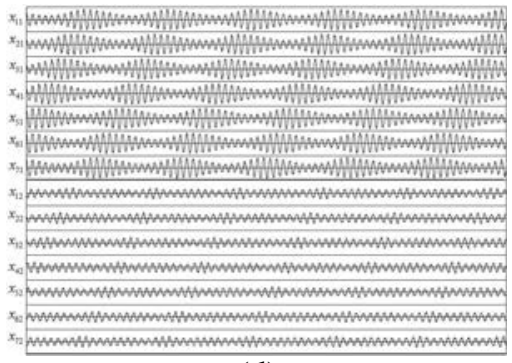

(d)

Figure 6: Asynchronous oscillations of double-mode and 7phase obtained from 7 coupled oscillators for $\alpha_{C}=0.8, \alpha_{L}=1.0$ $\gamma=0.3, \varepsilon=0.3$, and $\beta=3.5$. (a) Attractors observed at 14 resonators. (b) Phase relationship between horizontally adjacent resonators. (c) Phase relationship between two resonators in each oscillator. (d) Time waveforms of the voltages of 14 resonators

[7] M. Matsuki and S. Mori, "Asynchronous excitation phenomena in negative resistance oscillatory circuit containing periodically operating analog switch," IEICE Technical Report on NLP, Vol. 94, pp. 51-58, May 1994.

[8] Y. Nishio, Y. Yang and Y. Uwate, "Synchronization phenomena in simultaneous oscillators coupled by an inductor," Proceedings of NCSP'11, pp. 147150, Mar. 2011

[9] S. Fujioka, Y. Yang, Y. Uwate and Y. Nishio, "Double-mode simultaneous oscillation in three coupled hard oscillators," Proceedings of NCSP'12, pp. $72-75$, Mar. 2012.

[10] S. Fujioka, Y. Yang, Y. Uwate and Y. Nishio, "Two kinds of waves in a ladder of coupled simultaneous oscillators," Proceedings of NDES'12, pp. 232-236, Jul. 2012.

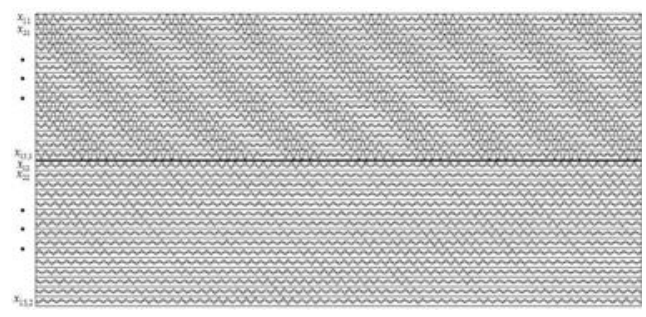

Figure 7: Asynchronous oscillations of double-mode and 15phase obtained from 15 coupled oscillators for $\alpha_{C}=0.8, \alpha_{L}=$ $1.0, \gamma=0.3, \varepsilon=0.3$, and $\beta=3.5$. 